\title{
Copeptin as an Inflammatory Marker in Diagnosis and Prognosis of Community Acquired Pneumonia in Children
}

A.S.Shebl, A.A.Sobieh, Y.I.Elsayed and M.R.Mohamed

Pediatric, Dept., Faculty of Medicine, Benha Univ., Benha, Egypt

E-Mail: M.R124@gmail.com

\section{Abstract}

A pertinent issue in the administration of pneumonic contaminations is the low explicitness of clinical side effects for the specific determination and the need of anti-infection treatment. A perfect biomarker for bacterial pneumonic diseases ought to permit a quick conclusion, have a prognostic esteem and encourage restorative dynamic.. The mix of a few biomarkers reflecting distinctive pathophysiological pathways can possibly improve the administration of network gained pneumonia later on.

\section{Introduction}

Pneumonia is the main sources of death around the world. Since pneumonia is normal and is related with huge dreariness and mortality, instantly diagnosing pneumonia, accurately perceiving any intricacies or fundamental conditions, and fittingly treating patients are exceedingly significant.

Pediatric pneumonia is answerable for the passings of in excess of 800,000 small kids worldwide every year, as indicated by the United Nations Children's Fund [1]. These passings happen solely in youngsters with hidden conditions, for example, ceaseless lung infection of rashness, inborn coronary illness, and immunosuppression [2] .

Network obtained pneumonia (CAP) is the most widely recognized conceivably deadly irresistible illness worldwide In CAP clinical science boundaries are utilized in routine diagnostics consistently for the determination of contamination and follow-up of the infection. Since CAP is an irresistible sickness, generally utilized research center qualities incorporate the white platelet check (WBC) and C-receptive protein (CRP). As of late biomarkers have been seriously concentrated in CAP, for the right finding of CAP as well as for diagnosing its microbiological etiology, seriousness of sickness, visualization and treatment decisions [3].

Copeptin is one of these new biomarkers.it is a Cterminal piece of pre-provasopressin which is an antecedent protein comprises of arginine vasopressin ,neurophysinII and copeptin [5].

These segments are isolated during axon transport from the phone body to the axon terminals in the back pituitary organ. On the way from the nerve center to the pituitary organ, copeptin and neurophysin II go about as transporter proteins of AVP. Copeptin is put away in the neurohypophyseal vesicles along with AVP and neurophysin II until they are discharged .. Copeptin is cosynthesized with AVP and is found in equimolar sums with AVP in the flow of sound and basically sick subjects. Along these lines copeptin mirrors AVP fixation and can be utilized as a substitution biomarker of AVP discharge [6].

copeptin may help in distinguishing patients with generally safe of death. Since it is autonomous of patient's age, it very well may be a significant prognostic factor in youthful patients with CAP [4].

\section{Subjects and methods}

2.1Subjects

\subsubsection{Study design}

This examination is a cross sectional investigation was carried on 50 youngsters experiencing pneumonia of various etiologies conceded in pediatric emergency unit Benha University Hospitals and in pediatric division at benha medical coverage. in the period from November 2019 to january 2020. This investigation was endorsed by the moral board of trustees of the Faculty of Medicine, Benha University.

Educated composed assents were taken from guardians of the included patients.

\section{I- Inclusion criteria}

All enrolled patients were:

- Admitted in pediatric ward and PICU aged between 1 month and 18 years old (both males and females).

- Children with diagnosis of pneumonia.

\section{II-Exclusion criteria}

Any patient was excluded from this study if:

- Children with any chronic chest illness other than pneumonia .

- patients with renal impairment.

- Patients with liver impairment.

- patients below one month and above 18 years.

\subsubsection{Study}

50 children aged from 2 months to 18 years with mean age $(54.32 \pm 39.53(4-120)$

They were 28 males (56\%) and 22 females $(44 \%)$.

Parental consent was granted in all cases and two children died after examination.

Their diagnosis based on clinical, laboratory, radiological evaluation.

\subsection{Methods}

All studied patients have been subjected to the following:

\section{Demographic data including:}

I- Full history taking in the form of applied questionnaire includin Sample

- Personal history: age, sex, residence.

- Onset, duration of illness.

- Presenting symptoms: fever, cough, tachypnea, cyanosis, recurrent attacks and congenital anomalies .

- Medication received

- Outcome 
II - Full clinical examination in the form of:-

- General examination

- General look

\section{Vital signs}

Temperature, heart rate, respiratory rate, blood pressure, capillary refill

\section{Oxygen saturation}

Anthropometric measurements

(Head circumference, weight, height)

- Systemic examination for

- Cardiac system

Inspection and palpation to detect the presence of pericardial bulge, pulsations and to examine apex. Auscultation: for heart sounds and audible murmur.

\section{Respiratory system}

- Inspection: to detect retractions, chest movements and signs of respiratory distress.

- palpation: for tracheal shift and palpable ronchi.

- Auscultation: for air entry, breath sounds and advential sound(fine crepitations and wheez)

Neurological examination

- Assessment of conscious level by Galscow coma scale.

- Examination of muscle power, tone and reflexes. Abdominal examination

- Inspection: for abdominal distention

- Palpation: for organomegaly (hepatomegaly and/ or splenomegaly)

- Percussion: for ascites.

- Auscultation: for intestinal sound

III - Investigations

Biochemical and hematological investigations: Measurement of

- Complete blood count.

- C-reactive protein.

- Serum copeptin.

1. Complete blood count (CBC) was done for all samples using sysmex KX-21N (Sysmex Corporation, New York, USA) for red blood cell (RBC) count, hemoglobin level, hematocrit value, WBC count (total and differential), and platelet count.

2. C- reactive protein

- Sample collection and storage: Fresh sample of venous blood was allowed to clot form and retract centrifuge clotted blood sample and collect serum, store at 2-8oC AVITEX-

- Quantitative measurement of the level of Creactive protein (CRP).

- Estimation was carried out using the test kit (Cromatest) at $0 \mathrm{hr}$ of clinical presentation. The AVITEX- CRP latex particles are coated with antibodies to human CRP. When the latex suspension is mixed with serum containing elevated CRP levels on a slide, clear agglutination was seen within 2 minutes.
- CRP had a detection limit of $6 \mathrm{mg} / \mathrm{L}$ of CRP in the patient's serum

\section{3- Serum copeptin by ELISA technique}

Sample collection and presentation: collect $5 \mathrm{ml}$ of venous blood aseptically. Allow the blood to coagulate at room temperature 10-20 minutes . centrifugation at the speed of 2000-3000r.p.m. and separate the supernatant.

\subsubsection{Principle}

The packs utilized adouble counter acting agent sandwich protein connected immunosorbent assay(ELISA) to test the degree of human copeptin in samples.copeptin was added to monoclonal immunizer catalyst which was precoated with human copeptin monoclonal neutralizer then brooding then copeptin antibodies marked with biotin were included and joined with streptavidin - HRP to frame safe complex at that point completed hatching and washing again to expel the uncombined enzyme.chromogen A,B was included .the shade of fluid chamged into blue and at the impact of corrosive ,the shading at last became yellow.the chroma of shading and the convergence of human substance copeptin of test were emphatically related.

\section{- Imaging}

All patients under went chest $x$-beam to affirm finding of pneumonia and to bar unfamiliar body goal

15 cases indicated peihilar and parabronchial penetrates of interstitial pneumonia ,23 cases demonstrated reciprocal cushioned cotton appearance of bronchopneumonia and 3 cases indicated thick mistiness influencing one portion of the lung and diafnosed as lobar pneumonia..

9 cases were analyzed as mycoplasma pneumonia.

Three radiographic examples were seen in these cases: peribronchial and perivascular interstitial invades, sketchy solidifications, and homogeneous acinar unions like ground glass. The lower fields of the lungs are frequently influenced, and extension of the hilar organs is normal.

\subsubsection{Statistical analysis}

The clinical data were recorded on a report form. These data were tabulated and analysed using the computer program SPSS (Statistical package for social science) version 20 to obtain:

\section{Descriptive data}

Descriptive statistics were calculated for the data in the form of:

1- Mean and standard deviation $( \pm S D)$. Median and inter-quartile range (IQR) for quantitative data.

2- Frequency and distribution for qualitative data.

In the statistical comparison between the different groups, the significance of difference was tested using one of the following tests after establishing their non normality by K-S test (One-Sample KolmogorovSmirnov Test) of normality.

3- Student's t-test and Mann-Whitney test:- Used to compare mean of two groups of quantitative data of parametric and non-parametric respectively 
4- ANOVA test (F value) and kruskal-wallis test:-Used to compare mean of more than two groups of quantitative data of parametric and non-parametric respectively.

Inter-group comparison of categorical data was performed by using chi square test $\left(X^{2}\right.$-value $)$ and fisher exact test (FET).

$$
\begin{gathered}
x^{2}=\frac{\sum(\text { observed }-\operatorname{expected})^{2}}{\text { Expected }} \\
\text { Expected }=\frac{\text { col.total } x \text { row total }}{\text { Grand total }}
\end{gathered}
$$

5- Correlation coefficient:- to find relationships between variables.

$\mathrm{P}$ value $<0.05$ was considered statistically significant $(*)$ while $>0.05$ statistically insignificant $\mathrm{P}$ value $<0.01$ was considered highly significant $(* *)$ in all analyses.

\section{rho $\rightarrow$ Spearman's correlation coefficient}

It evaluates the linear association between 2 quantitative variables (one is the independent variable $\mathrm{X}$, and the other is the dependent variable, $\mathrm{Y}$ ).

Value of " $r$ " ranges from -1 to 1

- $0=$ no linear correlation

- $1=$ perfect positive correlation

- $-1=$ perfect negative correlation
Positive $=$ increase in the independent variable leads to increase in the dependent variable.

Negative $=$ increase in the independent variable leads to decrease in the dependent variable.

\section{Boxplots}

A useful way of graphically representing the symmetry of data is the boxplot. This type of graph displays the median value by a horizontal bar surrounded by $50 \%$ of the scores shown within a box.

This $50 \%$ of scores falls between the 25 th and 75 percentile marks. The 25 th percentile is at the bottom of the box and the 75th percentile is at the top. The whiskers extending from both ends of the box show the highest and lowest values that are not outliers. Outliers are scores in the distribution that are more than 1.5 box-lengths from the 25 th or 75 th percentile, and they are displayed by a circle; those that are more than 4 box-lengths away are shown by an asterisk.

\section{Results}

50 hospitalized pneumonic patient from 2 months to 18 years were included.

Copeptin level was significantly higher among clinically unstable pneumonic patients with grade IV respiratory distress (median5.6) than patients with grade II (median 0.97) and patients with grade III (median2.46).

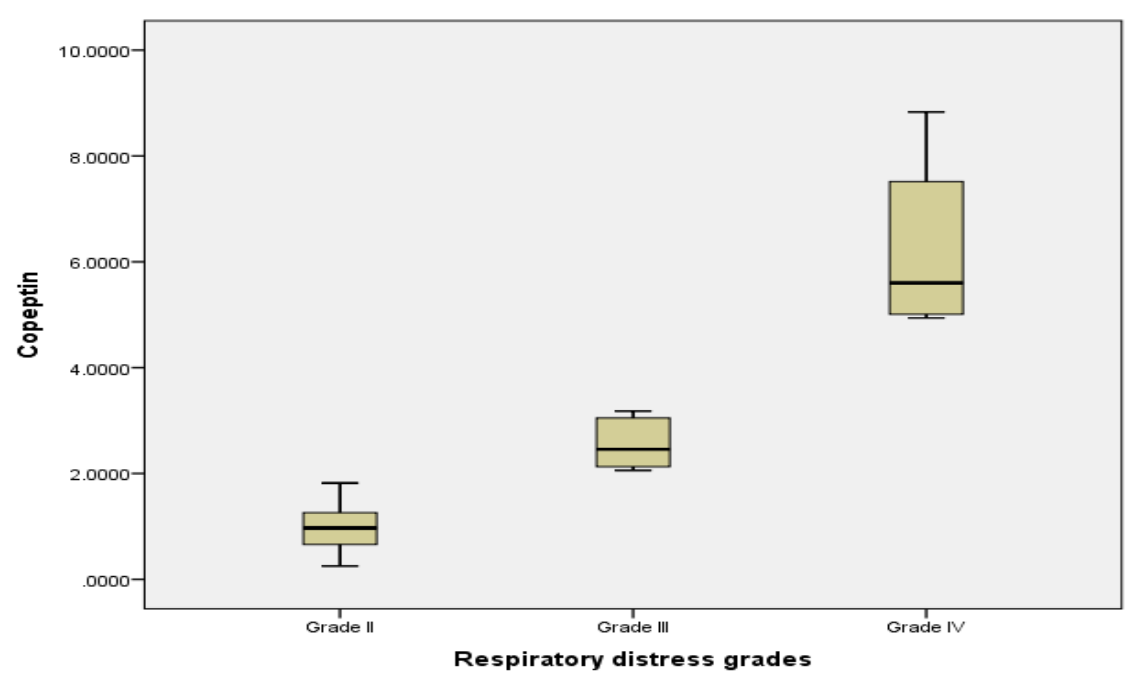

Fig (1) Boxplots show median and IQR between copeptin and respiratory distress grades.

Also, there was significant negative statistical correlation between age of studied cases and copeptin level in their serum so, infants had higher level of copeptin than old children SO they were at high risk of complications and high mortality ratio. 


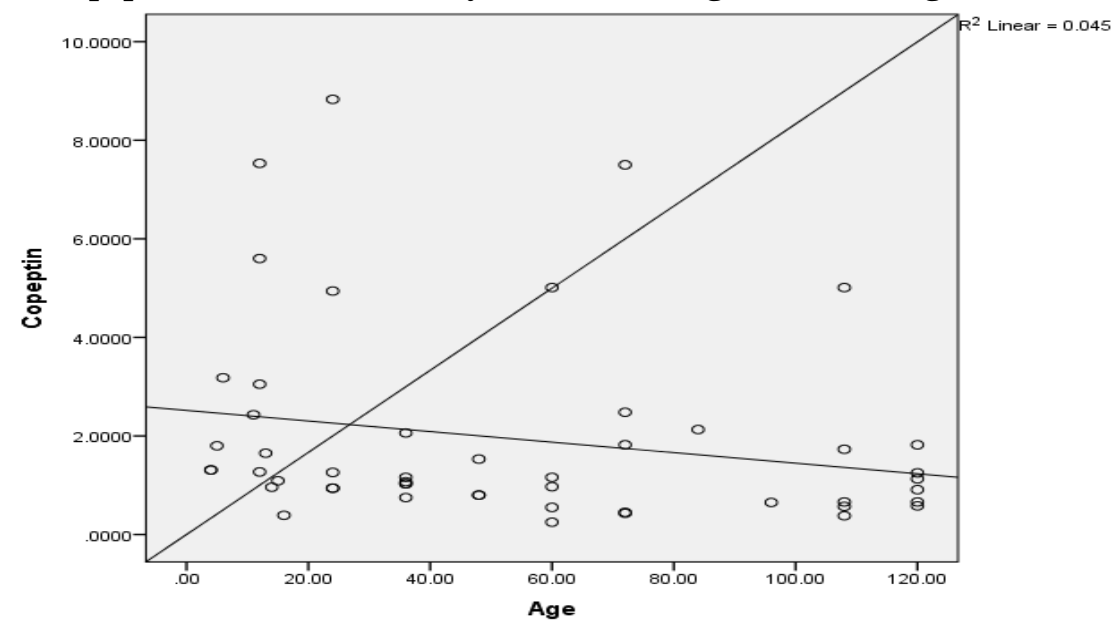

Fig (2) Scattered graph shows the relation between age and copeptin.

This study indicated that there was highly statistical significance in the correlation between respiratory distress and HB count in the studied patients with $\mathrm{p}$ value 0.04 . .

patients with grade II respiratory distress have higher HB count (median 10)than patients with RDIII(median 9.25) and patients with RDIV (Median 9). It means that HB count decreases with the severity of pneumonia .

It indicates bad prognosis of the disease and high mortality rate among these patients.

There were high levels of copeptin in patients who had low HB count which had significant statistical correlation. lower levels of $\mathrm{C}$ reactive protein in patients with pneumonia had significant statistical correlation with copeptin levels in the serum of the studied groups of patients.

This study showed that there was significant statistical correlation between copeptin and CRP with $\mathrm{p}$ value 0.028 .

CRP negative cases have higher level of copeptin(median 4.1) than CRP positive groups(median 1.16) .CRP negative cases were 5 patients and two of them were died after examination.

Table (1)The relation between respiratory distress grades and different parameters.

\begin{tabular}{|c|c|c|c|c|c|c|c|c|}
\hline \multirow{2}{*}{$\begin{array}{l}\text { Respiratory distress } \\
\text { grades }\end{array}$} & \multicolumn{2}{|c|}{ Grade II (37) } & \multicolumn{2}{|c|}{ Grade III (6) } & \multicolumn{2}{|c|}{ Grade IV (7) } & \multirow{2}{*}{$\begin{array}{c}\text { Statistical } \\
\text { test }\end{array}$} & \multirow[t]{2}{*}{$P$ value } \\
\hline & No & $\%$ & No & $\%$ & No & $\%$ & & \\
\hline Age & \multicolumn{2}{|c|}{$59.0 \pm 40.69$} & \multicolumn{2}{|c|}{$36.83 \pm 33.75$} & \multicolumn{2}{|c|}{$44.57 \pm 36.47$} & $\mathrm{~F}=1.06$ & 0.35 \\
\hline \multicolumn{9}{|l|}{ Mean \pm SD } \\
\hline Male & 22 & 59.5 & 4 & 66.7 & 2 & 28.6 & $\mathrm{FET}=2.5$ & 0.30 \\
\hline Female & 15 & 40.5 & 2 & 33.3 & 5 & 71.4 & & \\
\hline \multicolumn{9}{|l|}{ Residence } \\
\hline Urban & 8 & 21.6 & 0 & 0.0 & 1 & 14.3 & $\mathrm{FET}=1.17$ & 0.71 \\
\hline Rural & 29 & 78.4 & 6 & 100 & 6 & 85.7 & & \\
\hline $\begin{array}{l}\text { CBC for TLC count } \\
\text { Median (IQR) }\end{array}$ & \multicolumn{2}{|c|}{$\begin{array}{c}13000 \\
(11000-13000)\end{array}$} & \multicolumn{2}{|c|}{12500} & \multicolumn{2}{|c|}{14000} & $\mathrm{KW}=3.7$ & 0.16 \\
\hline \multicolumn{5}{|l|}{ Median (IQR) } & & & & \\
\hline $\begin{array}{l}\text { CRP Median } \\
\text { (IQR) }\end{array}$ & \multicolumn{2}{|c|}{$36.0(27.0-38.0)$} & \multicolumn{2}{|c|}{$37.0(33.5-43.5)$} & \multicolumn{2}{|c|}{$38.0(36.048 .0)$} & $\mathrm{KW}=2.79$ & 0.25 \\
\hline Positive & 36 & 97.3 & 6 & 100 & 6 & 85.7 & $\mathrm{FET}=2.52$ & 0.46 \\
\hline Negative & 1 & 2.7 & 0 & 0.0 & 1 & 14.3 & & \\
\hline $\begin{array}{l}\text { Copeptin Median } \\
\text { (IQR) }\end{array}$ & \multicolumn{2}{|c|}{$0.97(0.66-1.27)$} & \multicolumn{2}{|c|}{$2.46(2.11-3.08)$} & \multicolumn{2}{|c|}{$5.6(5.01-7.53)$} & $\mathrm{KW}=28.95$ & $<0.001 * *$ \\
\hline
\end{tabular}




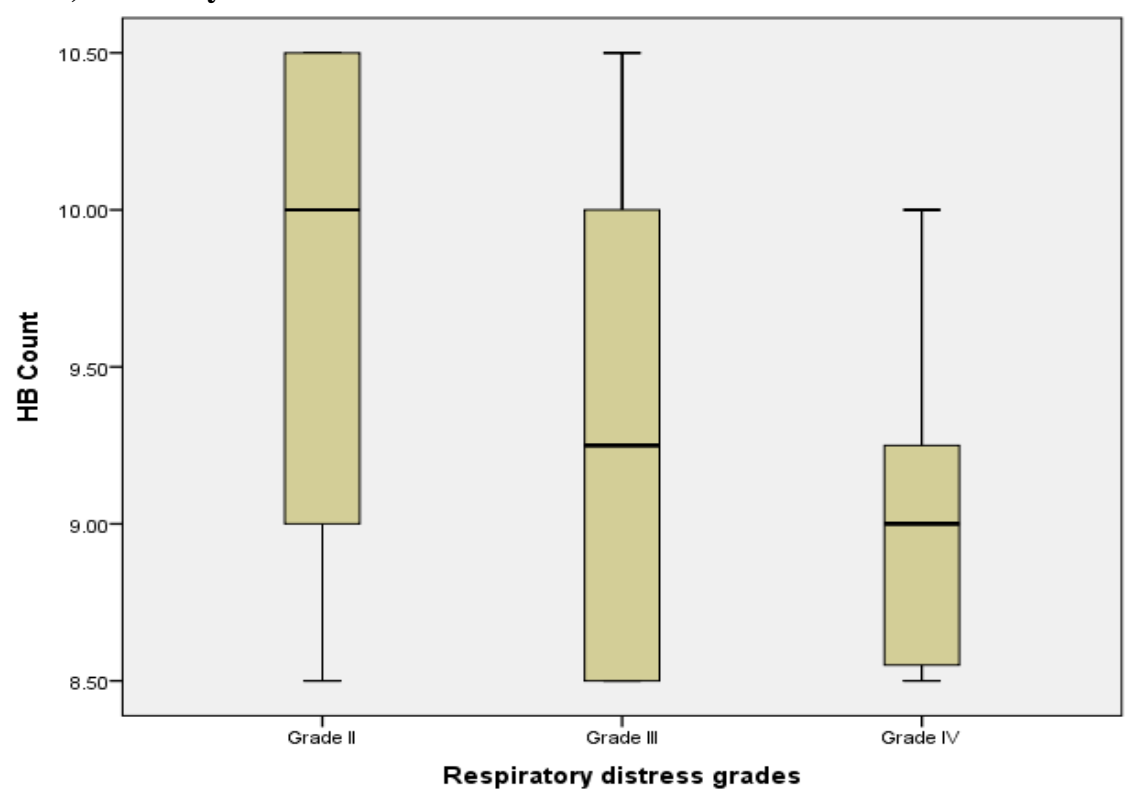

Fig (3) Boxplots show median and IQR between HB count and respiratory distress grades.

Table (2) The relation between copeptin and CRP.

\begin{tabular}{lccccc}
\hline \multicolumn{1}{c}{ Copeptin } & N & Median & IQR & $\begin{array}{c}\text { Statistical } \\
\text { test }\end{array}$ & P value \\
\hline $\begin{array}{l}\text { CRP } \\
\text { Positive }\end{array}$ & 45 & 1.16 & $0.80-2.0$ & MW $=0.42$ & $0.028^{*}$ \\
Negative & 5 & 4.1 & $0.66-4.1$ & & \\
\hline
\end{tabular}

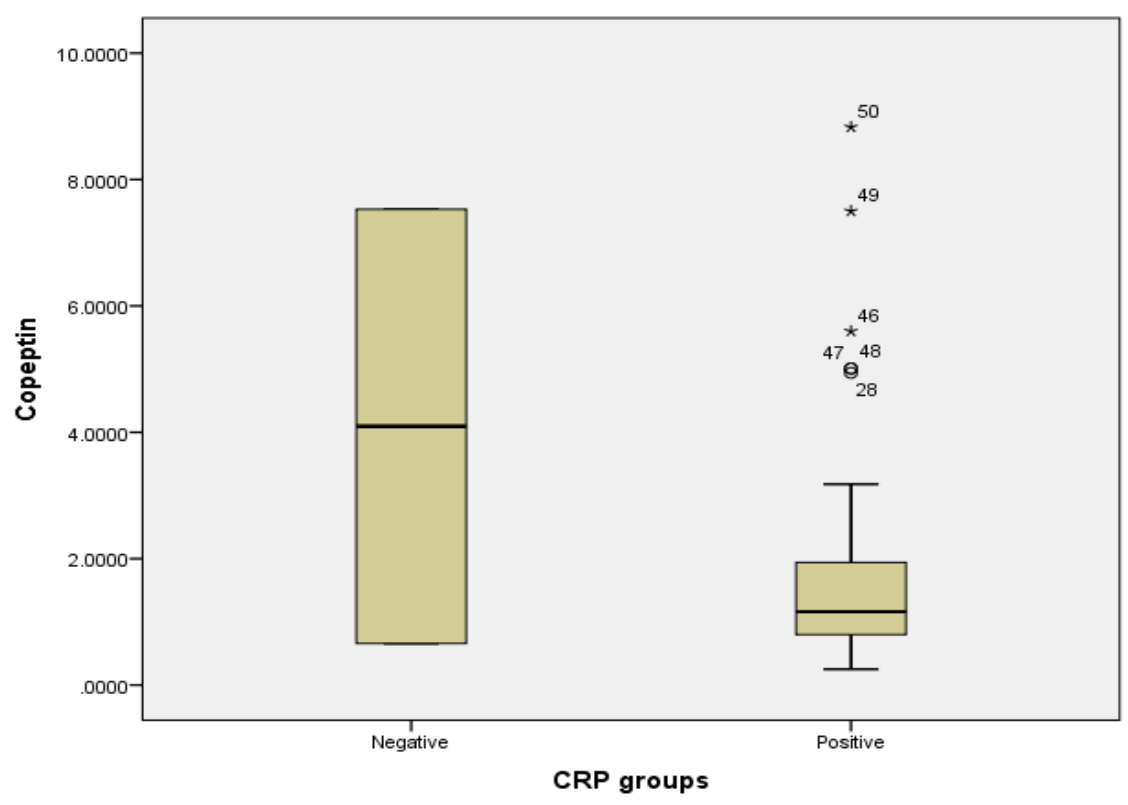

Fig (4) Boxplots show median and IQR between copeptin and CRP group.

In This current study showed that, there was significant correlation between respiratory distress grades and TLC count with p value 0.048 .

patients with respiratory Distress grade IV had higher level of TLC(14000 (13000- 15000) than other levels (gradeIII12500and grade II13000 ) leucocytosis in our study was indicative of mortality and poor outcome of pneumonia. 


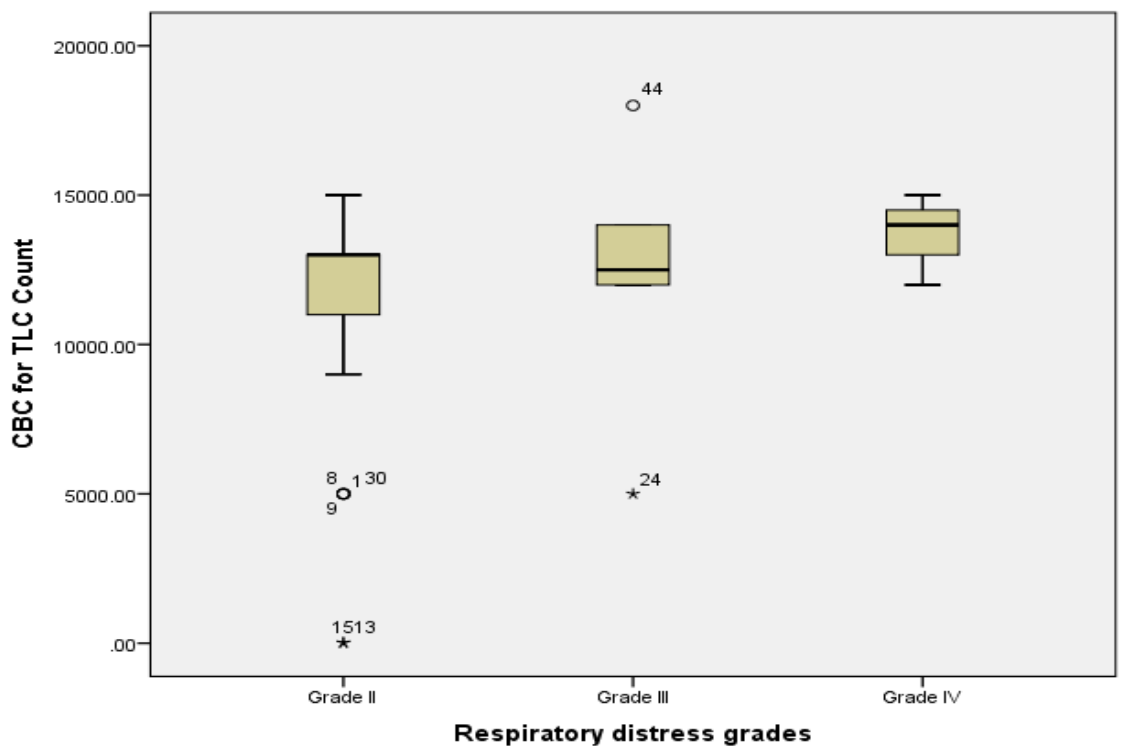

Fig (5) Boxplots show median and IQR between CBC for TLC count and respiratory distress grades.

\section{Discussion}

Network gained pneumonia in youngsters under 5 years old is the significant reason for mortality. It is answerable for the passings of more than $(800 \mathrm{~K})$ small kids worldwide every year as indicated by the United Nations youngsters' store [1]. In late years there has been race to discover new biomarkers related with irritation ln network gained pneumonia. Copeptin is preferable mortality prescient marker over long standing provocative boundaries, for example, CRP or absolute leukocytic tally at affirmation [10]

Copeptin likewise demonstrated empowering signs as aprognostic marker in network gained pneumonia. This was proposed in audit by [15].

This examination intended to survey the likely job of copeptin with absolute leucocytic include and Cresponsive protein in analysis and visualization of network obtained pneumonia.

The current examination was directed on 50 newborn child and youngsters experiencing network obtained pneumonia of various etiologies conceded in pediatric emergency unit and pediatric ward. Their ages extended from 2 months to 18 years with mean age $(54.3 \pm 39.533$ months). They were 28 guys $(56 \%)$ and 22 females $(44 \%)$.

With respect to the determination of the considered cases it relied upon three fundamental indications: fever, hack and respiratory exertion (respiratory distress).Moderate level of fever (38.5-39.5 c) happened in $65 \%$ in females and $61.9 \%$ in guys. Hack happens more in country females (19 cases) than rustic guys (18 cases) yet it happens in urban guys (6cases) more than urban females (2cases).

Respiratory misery is the most significant sign in network obtained pneumonia in youngsters. It has four evaluations, grade I (tachypnea ), grade II(retraction) ,grade III (snorting) and grade IV(cyanosis).Grade II had higher percent $(70 \%)$ than different evaluations (grade III 19\% in guys and $10 \%$ in females, grade IV 9.5\%) According to the evaluation of respiratory pain we can decide whether the youngster should be conceded in PICU or in the pediatric ward. We additionally can decide whether the kid needs nasal 02 or mechanical ventilation from the evaluation of respiratory trouble, so this examination exhibited the relationships and measurably distinction between respiratory misery and various boundaries.

After the principal month of life, hack is the most widely recognized introducing manifestation of pneumonia. Babies may have a past filled with predecessor upper respiratory symptoms.Tachypnea, withdrawals, snorting and cyanosis are normal and might be joined by fever, touchiness, and diminished feeding [17] .

This investigation showed that there was profoundly measurable essentialness in the connection between's respiratory pain and $\mathrm{HB}$ include in the considered patients with $\mathrm{p}$ esteem 0.04 . .

patients with grade II respiratory trouble have higher HB tally (middle 10) than patients with RDIII(median 9.25) and patients with RDIV (Median 9) . It implies that $\mathrm{HB}$ tally diminishes with the seriousness of pneumonia . it shows terrible guess of the ailment and high death rate among these patients.

This comes in concurrence with, who showed a factually critical relationship between lower HB trigger and expanded in-emergency clinic mortality in patients hospitalized with pneumonia. An authentic associate investigation of all patients hospitalized in an inside medication ward somewhere in the range of 2009 and 2014 with pneumonia, who got in any event 1 unit of $\mathrm{RBC}$, was assessed. The essential result was all-cause in-medical clinic mortality.One hundred guys and 77 females with a middle age of 10 ( range 2 - 18) years 
were incorporated. The middle $\mathrm{Hb}$ trigger was 8.10 $\mathrm{g} / \mathrm{dL}$. Death rate was $56 \%$ in patients with $\mathrm{Hb}$ trigger $\leq 7 \mathrm{~g} / \mathrm{dL}, 43.8 \%$ in $\mathrm{Hb}$ trigger 7 to $8 \mathrm{~g} / \mathrm{dL}$, and $29.5 \%$ in $\mathrm{Hb}$ trigger $>8 \mathrm{~g} / \mathrm{dL}(\mathrm{P}=.045)$. Patients in the $3 \mathrm{Hb}$ trigger classes didn't vary in age, sex, comorbidities, egg whites, creatinine, C-responsive protein, white platelets, and platelet tallies. The consequence of a multivariate investigation demonstrated that lone lower $\mathrm{Hb}$ trigger (chances proportion $[\mathrm{OR}] \leq 7 \mathrm{vs} .>8=5.24$, OR7-8vs. $>8=2.13, \mathrm{P}=.035$ ) and higher neutrophil check $(\mathrm{P}=.012)$ were related with expanded inmedical clinic mortality.

In This current examination demonstrated that, there was huge relationship between's respiratory misery evaluations and TLC tally with p esteem 0.048.

patients with respiratory Distress grade IV had more elevated level of TLC(14000 (13000-15000) than different levels (gradeIII12500and grade II13000) leucocytosis in our investigation was characteristic of mortality and helpless result of pneumonia.

This outcome comes as per [21], [18] who found that there was an extensive proof that leukocytosis might be an autonomous indicator for death. An examination from Spain explored the relationship of the WCC to mortality among 152 kid who had demonstrated pneumonia .The absolute leukocyte consider contrasted fundamentally as a real part of the individuals who kicked the bucket, contrasted and the individuals who endure. The creators presumed that there was a relationship between expanded WCC and a shorter ensuing endurance time. $(\mathrm{P}=0.0001)$

As opposed to our investigation [14]reported, that patients with leukopenia face a high danger of mortality, better starting acknowledgment of these serious cases would permit quick organization of proper treatment. It was structured as a case-contextual investigation settled in an accomplice study. A sum of 148 instances of network gained pneumonia were incorporated. The accompanying information were gathered: essential segment data, clinical history, signs and indications, radiological discoveries and research center outcomes during the initial $48 \mathrm{~h}$ of hospitalization. The investigation populace was isolated into 2 gatherings: (1) with extreme leukopenia (leukocyte check $\leq 3,000$ leukocytes/mL, $\mathrm{n}=62$ ) and (2) without serious leukopenia $(>3,000$ leukocytes $/ \mathrm{mL}$, $\mathrm{n}=86$ ). The by and large in-emergency clinic death rate was $41.2 \%$. Passing happened in $75.8 \%$ of extreme leukopenia cases with middle endurance season of 4 days, and in $16.3 \%$ of cases with leukocyte check $>3,000 / \mathrm{mL}(\mathrm{P}<0.001)$.

This examination demonstrated that Serum copeptin level was altogether higher in Pneumonic patients with respiratory misery grade IV (median5.6) than grade III median2.46) and grade II (median0.97). The connection between's respiratory misery and copeptin in our examination had exceptionally factual importance with $\mathrm{p}$ esteem $<0.001$.

This comes in CM.Nascimento-Carvalho et al., [14] who announced that copeptin was known to mirror the seriousness of pneumonia and compared with confusions of pneumonia in youngsters.

373 successive patients with CAP and 50 sound controls were assessed. Serum copeptin levels were estimated with another chemiluminescent sandwich immunoassay, Copeptin levels were essentially higher in patients with LRTI when contrasted with controls ( $\mathrm{P}$ $<0.001)$ with most significant levels in patients with CAP. Copeptin levels expanded with expanding seriousness of CAP $(\mathrm{P}<0.001)$. In patients who passed on, copeptin levels on confirmation were essentially higher when contrasted with levels in survivors [70.0 (28.8-149.0) versus 24.3 (10.8-43.8) pmol L(- 1), P < $0.001]$.

Also, M.N.Tsolia et al., [13] reported that copeptin concentrations were significantly higher in PICU pneumonic patients . Hemodynamic, laboratory and clinical data were recorded daily during the first 7 days after intensive care unit or hospital admission. Parallel thereto, blood was withdrawn to determine plasma AVP (radioimmunoassay) and copeptin (immunoluminometric assay) concentrations. Standard tests, a mixed effects model, and a linear regression analysis were used for statistical analysis). patients with sever pneumonia and severe sepsis had higher AVP levels than other patients $\mathrm{p}<0.001$

IN Community Acquired Pneumonia, copeptin is a free indicator of this sickness, and may give extra data about the seriousness of the infection [20]

He directed a planned observational investigation of patients with CAP. He estimated distinctive biomarkers in serum tests acquired at conclusion and performed univariate and multivariate examinations to distinguish likely indicators of mortality.

copeptin fixations were estimated in 173 patients. He found a positive relationship between's pneumonia seriousness and copeptin level $\mathrm{P}<0.0001 .$. In multivariate investigation including procalcitonin, Cresponsive protein, ANP ,lipopolysacchariderestricting protein and copeptin focuses, just Copeptinp stayed a free indicator of death (chances proportion 1.05, $\mathrm{P}=0.007)$. Copeptin demonstrated the most elevated indicative exactness to foresee mortality [13]also found that Copeptin had a functioning job and was better than conventional fiery markers in network procured pneumonia as it was more steady and its level in serum of patients was not influenced with pressure.. He selected 728 youngster with CAP. Midregional proadrenomedullin (MR-proADM), midregional proatrial natriuretic peptide (MR-proANP), proargininvasopressin (copeptin), proendothelin-1 (CT-proET-1), procalcitonin (PCT), C-receptive protein, white platelet (WBC) tally, and clinical confusion, and respiratory rate were resolved on affirmation.

In patients who kicked the bucket, MR-proADM, MR-proANP, copeptin, CT-proET-1 and PCT were fundamentally higher contrasted and survivors. For expectation of mortality the $\mathrm{C}$ record of proargininvasopressin (copeptin (0.78) was higher than that for MR-proANP (0.74), CT-proET-1 (0.76), PCT, C- 
receptive protein, and white platelets. proargininvasopressin (copeptin was free of CRB-65, and included prognostic data for short-and long haul mortality

Likewise, Ewig S and (5) announced that Copeptin serum levels increment with an expanding seriousness of .they moved 370 hospitalized patients with demonstrated CAP. Venous blood tests were gathered at the hour of consideration into the investigation and at the earliest opportunity after the finding of CAP. Copeptin levels were resolved in venous blood on affirmation. Copeptin levels expanded with expanding seriousness of CAP in patients without anti-toxin pretreatment however not in patients with anti-microbial pre-treatment. Patients with earlier anti-microbial treatment indicated altogether lower levels of copeptin [median (interquartile extend): 12.8 (5.3-22.6) versus 20.8 (11.1-37.8) pmol/L, P < 0.0001]

In this examination we attempted to discover huge relationships between's degree of copeptin in serum of patients and various boundaries to favor the likely job of copeptin in guess of network procured pneumonia.

This examination demonstrated that there was noteworthy negative connection between's copeptin level in serum and period of cases which has critical factual worth ( $p$ value0.016) Serum copeptin level was higher in babies than old kids so ,they were at higher danger of confusions and mortality than old youngsters.

This comes in concurrence with who revealed that Copeptin may help in recognizing patients with law danger of death as it is autonomous of patients age, it very well may be significant prognostic factor in little youngsters with network procured pneumonia

$\mathrm{He}$ directed an imminent observational investigation of173 patients with CAP(age go from 6months to18years). We estimated biomarkers in serum tests acquired at finding and performed univariate and multivariate investigations to recognize likely indicators of mortality

copeptin and ANP fixations were estimated in 173 patients. We found a positive relationship between's period of patient and copeptin level in serum $\mathrm{P}$ $<0.0001$ )

This examination demonstrated that there was huge measurable relationship among's copeptin and CRP with $\mathrm{p}$ value 0.028 .

CRP negative cases have more elevated level of copeptin(median 4.1) than CRP positive groups(median 1.16) .CRP negative cases were 5 patients and two of them were kicked the bucket after assessment.

So,Patients with negative $\mathrm{C}$ responsive protein were at higher hazard for intricacies of pneumonia than patients with positive $C$ receptive protein.

CRP is an intense stage reactant whose level in the serum ascends with aggravation. It ascends inside 6 hours of the beginning of irritation and may ascend to 10,000 folds topping at 48 hours, and vanishes similarly quickly after the ruinous procedure has stopped. CRP isn't just valuable in deciding the nearness of an incendiary sickness but on the other hand is useful in following its encouraging and adequacy of treatment. Specifically sequential estimations of CRP is valuable, for example when one needs to choose about the length of anti-toxin treatment in disease [6].

This outcome comes as per [9] who Found lower levels of $\mathrm{C}$ receptive protein in patients with atypical network gained pneumonia which is a cut off pneumonia with un ideal result than average network obtained pneumonia. Clinically precarious patients with pneumonia had low CRP level at the underlying phase of the illness As the limit of CRP fixation is accomplished after just 48 hours.

An absolute 258 back to back patients matured $<14$ yrs with intense side effects reliable with CAP were contemplated

Clinical, research center and radiological highlights at introduction just as other epidemiological information were recorded on a particular survey and entered in a PC database. Mean CRP levels as per aetiological conclusion are appeared in this table.

Table (3)The Relation Between Crp Mean And Type Of Pneumonia.

\begin{tabular}{lcc}
\hline Agent & Patients n & CRP mean $^{\text {mg・ dL }} \mathbf{~}^{-1}$ \\
\hline Typical bacterial pneumonia & 106 & 16 \\
Streptococcus pneumonia & 80 & 17.15 \\
Haemophilus influenza & 26 & 12.93 \\
Atypical pneumonia & 52 & 12.64 \\
Chlamydia pneumonia & 20 & 11.74 \\
Mycoplasma pneumonia & 17 & 16.02 \\
Coxiella burnetii & 15 & 10.02 \\
Viral & 35 & 14.45 \\
\hline
\end{tabular}

CRP values (mean) were significantly higher in patients with typical CAP than in those withA typical CAP pneumonia and viral pneumonia $(\mathrm{p}=0.0002)$.
Patients with Atypical pneumonia and viral pneumonia had bad prognosis and were at higher risk of complications than typical CAP.

In contrast to our stady, J. Oosterheert(2008) demonstrated that daily measurements of serum CRP in 
the patients with severe CAP are useful for identifying the patients with a poor prognosis, and this biomarker is a better predictor than the commonly used markers of infection, such as body temperature and leukocyte count.

A total of 289 patients with severe CAP were enrolled in the trial. The patients' mean age was 13.8 yrs.. Slightly lower baseline CRP levels were observed in patients who had recieved outpatient antibiotic treatment $(\mathrm{p}=0.07)$. No significant association between baseline CRP levels and demographic characteristics or the presence of comorbidity was observed $(\mathrm{p}>0.25)$.

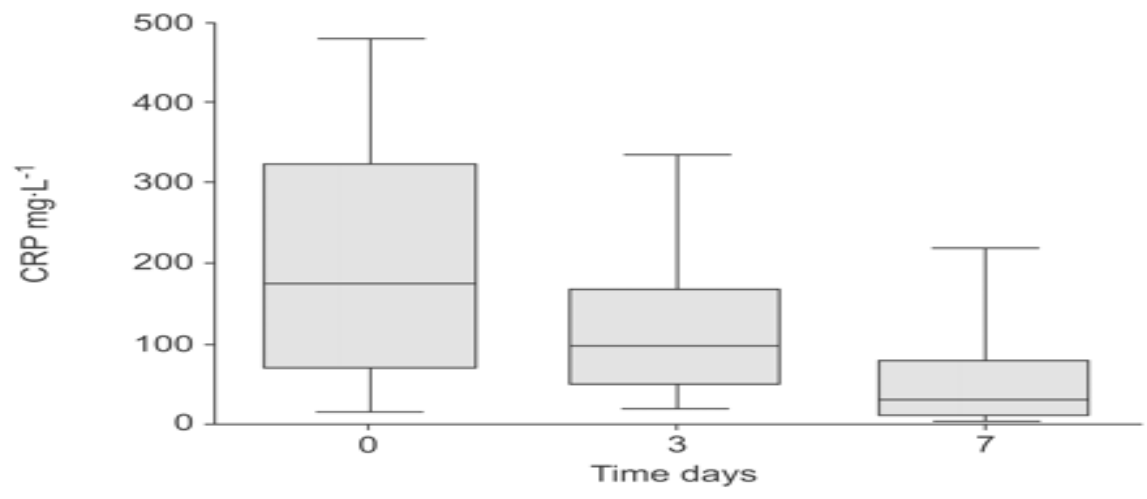

Fig (6) boxplots show median and IQR of CRP and time per days

Taking everything into account, back to back Creceptive protein estimations are helpful in the main week in follow-up of anti-infection treatment for serious network gained pneumonia,. A deferred decrease in C-receptive protein levels is related with a higher danger of having gotten improper anti-toxin treatment

This examination announced that copeptin was more solid in deciding anticipation of CAP in kids than other provocative markers .connections between's copeptin level ,CRP ,period of patients and HB include had apotential job in deciding patients who were in danger for confusions and mortality.

\section{Conclusion}

Copeptin level was fundamentally higher among clinically insecure pneumonic patients with grade IV respiratory pain (median5.6) than patients with grade II (middle 0.97) and patients with grade III(median2.46) .

Likewise, there was noteworthy negative factual connection between's period of contemplated cases and copeptin level in their serum along these lines, babies had more significant level of copeptin than old youngsters SO they were at high danger of inconveniences and high mortality proportion.

There were elevated levels of copeptin in patients who had low HB tally which had critical measurable relationship.

lower levels of $\mathrm{C}$ responsive protein in patients with pneumonia had critical factual relationship with copeptin levels in the serum of the considered gatherings of patients.

There was a significant proof that leukocytosis might be an autonomous indicator for terrible guess of the examined cases .

\section{References}

[1] UNICEF, Save the Children, and Every Breath Counts. Every child's right to survive: a 2020 agenda to end pneumonia deaths; Vol.22, PP.5259,2020 .

[2] I.Rudan, C.Boschi-Pinto, Z.Biloglav, K.Mulholland, H.Campbell,Epidemiology and etiology of childhood pneumonia; Vol.122, PP.259251.2008.

[3] M.WoodHead,Guidelines for the management of adult lower respiratory tract infections--full version;Vol.22, PP.221-226,2011.

[4] M.Masiá, J.Papassotiriou, N.G.Morgenthaler, I.Hernández, C.Shum, F.Gutiérrez, Midregional Pro-A-type natriuretic peptide and carboxyterminal provasopressin may predict prognosis in community-acquired pneumonia;Vol.26, PP.42-46, 2007.

[5] G.Szinnai, N.G.Morgenthaler, K.Berneis, J.Struck, B.Müller, U.Keller, M.Christ-Crain ,Changes in plasma copeptin, the c-terminal portion of arginine vasopressin during water deprivation and excess in healthy subjects;Vol.12, PP.92-97, 2007.

[6] S.Balanescu, P.Kopp, M.B.Gaskill, N.G .Morgenthaler, C.Schindler, J.Rutishauser,Correlation of plasma copeptin and vasopressin concentrations in hypo-, iso-, and hyperosmolar states;Vol.11, PP.252-256, 2011.

[7] V.P.Shah, M.G.Tunik, J.W.Tsung, Prospective evaluation of point-of-care ultrasonography for the diagnosis of pneumonia in children ;Vol.22,pp66$72,2013$.

[8] A.P.Metinko,Neonatal pulmonary host defense mechanisms. Polin RA, Fox WW, eds. Fetal and Neonatal Physiology;Vol.9, PP.221-224,2004.

[9] E.D.Barnett, J.O. Klein Bacterial infections of the respiratory tract. Remington JS, Klein JO, 
edsInfectious Diseases of the Fetus and Newborn Infant; Vol.17, PP.44-47, 2004.

[10] R.C.Bone, C.J.Grodzin, RA. Balk Sepsis: a new hypothesis for pathogenesis of the disease process; Vol.14, PP.82-88,2000.

[11] I.C.Michelow, K.Olsen, J.Lozano, Epidemiology and clinical characteristics of community-acquired pneumonia in hospitalized children;Vol.1, PP. 321-333, 2004.

[12] A.M.Mishaan, EO.Jr.Mason, G.MartinezAguilar, Emergence of a predominant clone of community-acquired Staphylococcus aureus among children;Vol.2, PP.90-96,2005.

[13] M.N.Tsolia, S.Psarras, A.Bossios, Etiology of community-acquired pneumonia in hospitalized school-age children: evidence for high prevalence of viral infection;Vol.10, PP.43-46,2004.

[14] CM.Nascimento-Carvalho, C.T.Ribeiro, M.R.Cardoso, The role of respiratory viral infections among children hospitalized for community-acquired pneumonia in a developing country;Vol.40, PP.223-228,2008.
[15] T.Juvén, J.Mertsola, M.Waris, Etiology of community-acquired pneumonia in 254 hospitalized children;Vol.22, PP.445-452,2000.

[16] J.Tajima Thomson, M.Hall, L.Ambroggio, Aspiration and non-aspiration pneumonia in hospitalized children with neurologic impairment;Vol.22, PP.54-58,2016.

[17] E.T.Nakayama, Y.Kondo , Etiology and clinical study of community-acquired pneumonia in 157 hospitalized children;Vol.12, PP.221-225,2006.

[18] W.W.Thompson, D.K.Shay, E.Weintraub, xInfluenza-associated hospitalizations in the United States;Vol.3, PP.441-447, 2006.

[19] S.T.Li, D.J.Tancredi , Empyema hospitalizations increased in US children despite pneumococcal conjugate vaccine;Vol.5, PP.114$122,2010$.

[20] I.Rudan, L.Tomaskovic, C.Boschi-Pinto, H.Campbell, Global estimate of the incidence of clinical pneumonia among children under five years of age;Vol.25, PP.141-144, 2004.

[21] S.Shah, R.Bachur, D.Kim, M.I.Neuman Lack of predictive value of tachypnea in the diagnosis of pneumonia in children;Vol.22, PP.79-82,2010. 